\title{
COINCIDENCE INDEX AND MULTIPLICITY
}

\author{
BY
}

\section{B. LALOUX AND J. MAWHIN}

\begin{abstract}
This paper is devoted to the extension, in the frame of coincidence degree theory in normed spaces, of the concept of Leray-Schauder index of an isolated fixed point. The generalization includes basic properties of the coincidence index, Krasnosel'skil type theorems for the case of noninvertible linear part and a Leray-Schauder's type formula relating the index and spectral theory in the linear case. This last problem needs the introduction of the concept of characteristic value for some couples of linear mappings and of its multiplicity.
\end{abstract}

1. Introduction. The concept of coincidence degree for couples $(L, N)$ in locally convex spaces, with $L$ a Fredholm linear mapping and $N$ some nonlinear mapping, has been recently introduced in [9]. When the Fredholm index of $L$ is equal to zero, the only case we shall consider here, this coincidence degree is an integer which reduces to the Leray-Schauder degree of $I-N$ if $L$ is the identity mapping $I$ and $N$ is completely continuous. Moreover the coincidence degree theory appears to be a convenient frame for studying various types of equations of the form $L x=N x$ when $L^{-1}$ does not exist [4] , [6] , [11] - [13] , [17] , in the same way that Leray-Schauder's degree is extremely useful for considering cases where $L$ is invertible.

On the other hand, it already appears in the fundamental paper [7] of Leray and Schauder that a basic tool for calculating the degree is the study of the index of an isolated fixed point of a completely continuous mapping. Leray and Schauder have in particular studied this index for one-to-one, for differentiable and for linear mappings, and, in this last case, they have shown that the index at zero of the invertible mapping $I-A$, with $A$ compact, is equal to $(-1)^{m}$, with $m$ the sum of the multiplicities of the characteristic values of $A$ lying in ]0, 1[. Later on, Krasnosel'skii [5] has given an important contribution to this type of problem by calculating the index of some completely continuous perturbations of identity whose linear part is not invertible and by showing the extreme importance of the concept of index in bifurcation theory. Further results in the line of Krasnosel'skii

Received by the editors November 27, 1973 and, in revised form, October 28, 1974. AMS (MOS) subject classifications (1970). Primary 47H15, 54H25, 55C25; Secondary 47A10, 47B05.

Key words and phrases. Coincidence degree, coincidence index, characteristic value, multiplicity, Leray-Schauder theory, bifurcation theory. 
are due to Melamed [14] - [15], Zabreiko and Krasnosel'skii [19].

The aim of this paper is to generalize this material from Leray-Schauder degree to coincidence degree of mappings in normed spaces. If some of those extensions, mostly the ones of $\$ 2$, are nearly trivial, other ones require more ingenuity and work. It is the case for the Krasnosel'skii type theorems of $\S 3$, which find a particularly natural setting in the frame of coincidence degree theory and that we prove in a simple new way. It is still more the case for the generalization of Leray-Schauder's formula for the index of linear compact perturbations of identity, which has needed a definition and a careful study of the concept of characteristic value for the couple $(L, A)$, with $L$ as above and $A$ linear, and of its multiplicity. As shown in $\$ 4$, a "nondegeneracy" assumption on $(L, A)$, trivially satisfied when $L=I$, has been revealed as crucial in defining the multiplicity and has made it possible to associate with the generalized characteristic value problem a classical one. Those results are then used in $\$ 5$ to prove a generalized Leray-Schauder formula relating the coincidence index of $(L, A)$, with $A$ linear and satisfying the "nondegeneracy" assumption, to the multiplicities of the generalized characteristic values.

Beyond their own interest, the results proved in this paper will be fundamental in developing the bifurcation theory based upon coincidence degree and initiated in [6].

2. The concept of coincidence index. Let $X, Z$ be normed real vector spaces, $a \in X$ and $\Omega$ a bounded open neighbourhood of $a$. Let $L: \operatorname{dom} L \subset X \rightarrow$ $Z$ be a (not necessarily continuous) linear mapping and $N: \Omega \rightarrow Z$ a (not necessarily linear) continuous mapping such that $N(\Omega)$ is bounded, which satisfy the following assumptions.

$\left(\mathrm{H}_{1}\right) L$ is a Fredholm mapping of index zero, i.e. $\operatorname{dim} \operatorname{ker} L=\operatorname{codim} \operatorname{Im} L$ $<\infty$ and $\operatorname{Im} L$ is closed in $Z$.

$\left(\mathrm{H}_{2}\right) L_{P}^{-1}(I-Q) N$ is completely continuous on $\Omega$, where $P, Q$ are continuous projectors such that the sequence

$$
X \stackrel{P}{\rightarrow} \operatorname{dom} L \stackrel{L}{\rightarrow} Z \stackrel{Q}{\longrightarrow} Z
$$

is exact at $\operatorname{dom} L$ and $Z$ [1] and $L_{P}$ is the restriction $L \mid(\operatorname{ker} P \cap \operatorname{dom} L)$ of $L$ at ker $P \cap \operatorname{dom} L$. The existence of $P$ and $Q$ is insured by assumption $\left(\mathrm{H}_{1}\right)$ and it is proved in [9] that condition $\left(\mathrm{H}_{2}\right)$ does not depend upon the choice of $P$ and Q.

$\left(\mathrm{H}_{3}\right)$ The point $a$ is an isolated zero of $L-N$.

It follows from assumption $\left(\mathrm{H}_{3}\right)$ that there exists an $\epsilon_{0}>0$ such that the closure $\mathrm{cl} B_{\epsilon_{0}}(a)$ of the open ball $B_{\epsilon_{0}}(a)$ of center $a$ and radius $\epsilon_{0}$ is contained in $\Omega$ and such that 


$$
\left\{(L-N)^{-1}(0)\right\} \cap \mathrm{cl} B_{\epsilon_{0}}(a)=\{0\} .
$$

Hence, for every $\epsilon \in] 0, \epsilon_{0}[$,

$$
0 \notin(L-N)\left(\operatorname{dom} L \cap \text { bdry } B_{\epsilon}(a)\right)
$$

and it follows then from [9] that the $L_{+}$-coincidence degree $d\left[(L, N), B_{\epsilon}(a)\right]$ of $L$ and $N$ in $B_{\epsilon}(a)$ will be defined and will not depend upon $\epsilon$. Let us recall that $d\left[(L, N), B_{\epsilon}(a)\right]$, that we shall call here simply the coincidence degree of $L$ and $N$ in $B_{\epsilon}(a)$, is defined in [9] as the Leray-Schauder degree [7] $d_{L S}\left[I-M_{\Lambda}, B_{\epsilon}(a), 0\right]$ with

$$
M_{\Lambda}=P+\Lambda \Pi N+L_{P}^{-1}(I-Q) N,
$$

II: $Z \rightarrow \operatorname{coker} L$ is the canonical surjection and $\Lambda: \operatorname{coker} L \rightarrow \operatorname{ker} L$ is an isomorphism preserving orientation chosen upon $\operatorname{ker} L$ and coker $L$. The relation of this coincidence degree with other generalized Leray-Schauder degrees has already been discussed in [9] but the following remarks which we owe to the referee are of special value in that they allow simplifications in some of the original proofs.

If $\Lambda_{Q}=\Lambda \Pi \mid \operatorname{Im} Q$, then

$$
M_{\Lambda}=P+\Lambda_{Q} Q N+L_{P}^{-1}(I-Q) N=(L+F)^{-1}(F+N)
$$

where $F=\Lambda_{Q}^{-1} P$, as it is easily checked by solving the equation $(L+F) x=y$ written in the equivalent form

$$
L_{P}(I-P) x=(I-Q) y, \quad \Lambda_{Q}^{-1} P x=Q y .
$$

Let us also note that $(L+F)^{-1} F=\Lambda_{Q} Q \Lambda_{Q}^{-1} P=P$. So

$$
d\left[(L, N), B_{\epsilon}(a)\right]=d_{L S}\left[I-(L+F)^{-1}(F+N), B_{\epsilon}(a), 0\right]
$$

if, in $F, \Lambda_{Q}$ corresponds to an orientation preserving $\Lambda$. In the case where $L$ is continuous, $L+F$ is a linear homeomorphism and the right-hand member of (2.1) is equal to the Browder degree [20] $\operatorname{deg}\left([G, S], B_{\epsilon}(a), 0\right)$ where $G=L-$ $N$ and $S: B_{\epsilon}(a) \times X \rightarrow Z$ is defined by

$$
S(x, v)=(L+F) v-(F+N) x .
$$

$S$ is called a representation of $G$ and it is proved in [20] that $\left|\operatorname{deg}\left([G, S], B_{e}(a), 0\right)\right|$ is independent of the representation, i.e. of $F=\Lambda_{Q}^{-1} P$. The basic results of the coincidence degree theory given in [9] explicit completely the dependence of the degree with respect to the choice of $F$.

The invariance of $d\left[(L, N), B_{\epsilon}(a)\right]$ with respect to $\epsilon$ justifies the following

DEFinition 2.1. Assumptions $\left(\mathrm{H}_{1}\right)-\left(\mathrm{H}_{3}\right)$ being supposedly satisfied, the coincidence index $i[(L, N), a]$ of $L$ and $N$ at point $a$ is the integer $d\left[(L, N), B_{\epsilon}(a)\right]$ for any $\left.\epsilon \in] 0, \epsilon_{0}\right]$. 
It follows at once from Definition 2.1 that

$$
i[(L, N), a]=i_{L S}\left[I-M_{\Lambda}, a\right]=i_{L S}\left[I-(L+F)^{-1}(F+N), a\right]
$$

where $i_{L S}$ denotes the Leray-Schauder index [7].

The interest of the coincidence index in computing the coincidence degree follows from the immediate consequence of above definition and corresponding result in Leray-Schauder theory given below.

Proposition 2.1. Let $L: \operatorname{dom} L \subset X \rightarrow Z, N: \mathrm{cl} \Sigma \rightarrow Z$ be mappings satisfying conditions $\left(\mathrm{H}_{1}\right)-\left(\mathrm{H}_{2}\right)$ above with $\Omega$ replaced by the open bounded subset $\Sigma$ of $X$. If

$$
0 \notin(L-N)(\operatorname{dom} L \cap \text { bdry } \Sigma)
$$

and if $(L-N)^{-1}(0)$ is a finite set $\left\{a_{1}, \ldots, a_{m}\right\}$, then

$$
d[(L, N), \Sigma]=\sum_{j=1}^{m} i\left[(L, N), a_{j}\right]
$$

3. Some results about the computation of $i[(L, N), 0]$. We shall be interested in this section in founding various conditions under which the coincidence index of $L$ and $N$ at some point, say the origin, can be estimated. The results we shall prove parallel, in the frame of coincidence index theory, some theorems of Leray and Schauder [7] and Krasnosel'skii [5] for completely continuous perturbations of identity. In the whole section, $\Omega \subset X$ will be an open bounded neighbourhood of the origin.

THEOREM 3.1. Let $L$ and $N$ satisfy assumptions $\left(\mathrm{H}_{1}\right)-\left(\mathrm{H}_{2}\right)$ above with $N(0)=0$ and let $L-N$ be one-to-one on $\Omega$. Then $i[(L, N), 0]$ exists and

$$
|i[(L, N), 0]|=1 \text {. }
$$

Proof. Let $B_{\epsilon}(0)$ be such that $\mathrm{cl} B_{\epsilon}(0) \subset \Omega$. It is clear that $(L-N)^{-1}(0)$ $=\{0\}$ and that $i[(L, N), 0]$ exists. By Theorem 3.1 of [9], $I-M_{\Lambda}$ is one-to-one on $\mathrm{cl} B_{\epsilon}(0)$ and hence, by a well-known theorem of Leray [8] (see also Browder [2] ), $d_{L S}\left[I-M_{\Lambda}, B_{\epsilon}(0), 0\right]= \pm 1$ which implies (3.1).

A more precise result will now be given, which needs more assumptions upon $N$. Its proof uses a lemma, first proved by the first author in a slightly different setting in [6], and that we shall give here explicitly for reader's convenience.

LEMmA 3.1. Suppose that $L$ satisfies assumption $\left(\mathrm{H}_{1}\right)$ and that $N=A+$ $B$ with $A: X \rightarrow Z$ linear, continuous, such that $L_{P}^{-1}(I-Q) A$ is compact and $\operatorname{ker}(L-A)=\{0\}$, and with $B: \Omega \rightarrow Z$ continuous and such that $L_{P}^{-1}(I-Q) B$ is completely continuous on $\Omega$. Then $(L-A): \operatorname{dom} L \rightarrow Z$ is onto, $(L-A)^{-1} B$ 
is completely continuous on $\Omega$ and, for every $x \in \Omega$,

$$
\left(I-M_{\Lambda}\right) x=\left(I-M_{\Lambda}^{*}\right)\left[I-(L-A)^{-1} B\right] x
$$

with

$$
M_{\Lambda}^{*}=P+\Lambda \Pi A+L_{P}^{-1}(I-Q) A .
$$

Proof. From Theorem 3.1 of [9] we get at once that $\operatorname{ker}\left[I-M_{\Lambda}^{*}\right]=\{0\}$ and hence, using Riesz theory [16], $I-M_{\Lambda}^{*}$ will be a linear homeomorphism of $X$, and hence of dom $L$ because of the form of $I-M_{\Lambda}^{*}$. Moreover, using Proposition 3.3 of [9],

$$
\left(I-M_{\Lambda}^{*}\right) x=\left[\Lambda \Pi+L_{P}^{-1}(I-Q)\right](L-A) x
$$

for each $x \in \operatorname{dom} L$ and hence, $\Lambda \Pi+L_{p}^{-1}(I-Q)$ being an algebraic isomorphism between $Z$ and dom $L$, we have

$$
(L-A) x=\left[\Lambda \Pi+L_{P}^{-1}(I-Q)\right]^{-1}\left(I-M_{\Lambda}^{*}\right) x
$$

for each $x \in \operatorname{dom} L$. Therefore $L-A$ is onto and, for each $z \in Z$.

$$
(L-A)^{-1} z=\left(I-M_{\Lambda}^{*}\right)^{-1}\left[\Lambda \Pi+L_{P}^{-1}(I-Q)\right] z,
$$

which clearly shows moreover that $(L-A)^{-1} B$ is completely continuous on $\Omega$. Relation (3.2) then follows from (3.3) by direct computation.

The following theorem extends to coincidence degree theory a classical result of Leray and Schauder [7].

THEOREM 3.2. Suppose that $L$ satisfies assumption $\left(\mathrm{H}_{1}\right)$ and that $N=A$ $+B$ with $A$ and $B$ as in Lemma 3.1. If

$$
\|Q B x\| \leqslant q_{1}(\rho)\|x\|, \quad\left\|L_{P}^{-1}(I-Q) B x\right\| \leqslant q_{2}(\rho)\|x\|,
$$

for every $x \in \Omega \cap B_{\rho}(0), \rho \in R_{+}$, with $q_{i}: R_{+} \rightarrow R_{+}$such that $\lim _{\rho \rightarrow 0+} q_{i}(\rho)$ $=0, i=1,2$, then condition $\left(\mathrm{H}_{3}\right)$ is satisfied and

$$
i[(L, N), 0]=i[(L, A), 0] \text {. }
$$

Proof. First, using Lemma 3.1, we have

$$
\left(I-M_{\Lambda}\right) x=\left(I-M_{\Lambda}^{*}\right)\left[I-(L-A)^{-1} B\right] x
$$

for every $x \in \Omega$, with $(L-A)^{-1} B$ completely continuous in $\Omega$. Now, by (3.3), (3.4), there exists $\epsilon_{1}>0$ such that $\operatorname{cl} B_{\epsilon_{1}}(0) \subset \Omega$ and $\left\|(L-A)^{-1} B x\right\| \leqslant(1 / 2)\|x\|$ for every $x \in \operatorname{cl} B_{\epsilon_{1}}(0)$. Hence, for every $\left.\left.\epsilon \in\right] 0, \epsilon_{1}\right]$ and every $(x, \lambda) \in$ cl $B_{\epsilon}(0) \times[0,1]$, we have

$$
\left\|x-\lambda(L-A)^{-1} B x\right\| \geqslant(1 / 2)\|x\|
$$

which first shows that $\left(\mathrm{H}_{3}\right)$ is satisfied and then, using the invariance of degree 
with respect to homotopy, that

$$
d_{L S}\left[I-\lambda(L-A)^{-1} B, B_{\epsilon}(0), 0\right]=1
$$

for every $\lambda \in[0,1]$. Now, using (3.6), (3.7) and Leray's product theorem [8] we obtain

$$
i[(L, N), 0]=d_{L S}\left[I-M_{\Lambda}, B_{\epsilon}(0), 0\right]=d_{L S}\left[I-M_{\Lambda}^{*}, B_{\epsilon}(0), 0\right]=i[(L, A), 0]
$$

which achieves the proof.

This theorem shows now the interest of computing $i[(L, A), 0]$ when $A$ is linear and $\operatorname{ker}(L-A)=\{0\}$. If $X=Z, L=I$ and $A$ is compact, a famous formula of Leray and Schauder [7] implies that $i_{L S}(I-A, 0)$ is equal to $(-1)^{\gamma}$, where $\boldsymbol{\gamma}$ is the sum of the multiplicities of the characteristic values of $\boldsymbol{A}$ belonging to ] 0,1 [. The two last sections of this paper will be devoted to the extension of this result to coincidence index.

We shall now be interested in computing $i[(L, N), 0]$ when the linear part of $L-N$ has a nontrivial kernel. Basic for this purpose are the two following lemmas.

LEMMA 3.2. Let $L, N$ satisfy assumptions $\left(\mathrm{H}_{1}\right)-\left(\mathrm{H}_{2}\right)$ and $N$ be of the form $N=H+R$ with $H: X \rightarrow Z$ continuous and homogeneous of degree $k>1$, such that

$$
\|Q H x\| \leqslant p_{1}(\rho)\|x\|, \quad\left\|L_{P}^{-1}(I-Q) H x\right\| \leqslant p_{2}(\rho)\|x\|,
$$

for every $x \in \mathrm{cl} B_{\rho}(0)$ and

$$
H x \notin \operatorname{Im} L \quad \text { for every } x \in \text { bdry } B_{1}(0) \cap \operatorname{Ker} L,
$$

and with $R: \Omega \rightarrow Z$ such that

$$
\|Q R x\| \leqslant q_{1}(\rho)\|x\|^{k}, \quad\left\|L_{P}^{-1}(I-Q) R x\right\| \leqslant q_{2}(\rho)\|x\|^{k}
$$

for every $x \in \Omega \cap \mathrm{cl} B_{\rho}(0)$, the functions $p, q: R_{+} \rightarrow R_{+}$going to zero when $\rho \rightarrow 0+$. Then condition $\left(\mathrm{H}_{3}\right)$ is satisfied and

$$
i[(L, N), 0]=i_{B}[-(\Lambda \Pi H) \mid \operatorname{ker} L, 0]=i_{B}[-(J Q H) \mid \operatorname{ker} L, 0],
$$

where $J=(\Lambda I) \mid \operatorname{Im} Q$ and $i_{B}$ denotes the Brouwer index.

Proof. For every nonzero $x \in \Omega$ we have

$$
\begin{aligned}
\left(I-M_{\Lambda}\right) x & =\left[I-P+\|x\|^{k-1} P\right]\left[(I-P) x-\|x\|^{1-k} \Lambda \Pi(H+R) x\right. \\
& \left.-L_{P}^{-1}(I-Q)(H+R) x\right] \\
& =\left(I-M_{1}\right)\left(I-M_{2}\right) x
\end{aligned}
$$

where $M_{1}$ is clearly completely continuous on every bounded set of $X$ and where $M_{2}$ is obviously extended to a completely continuous mapping on $\Omega$ by writing 
$M_{2}(0)=0$. Now, $I-P+\|\cdot\|^{k-1} P$ vanishes only at zero and hence, for each $\epsilon>0$ such that $\mathrm{cl} B_{\epsilon}(0) \subset \Omega$, we have

$$
\begin{aligned}
\inf _{x \in \operatorname{bdry} B_{\epsilon}(0)}\|(I-P) x-\| x\left\|^{k-1} \Lambda \Pi H x\right\| \\
=\inf _{x \in \operatorname{bdry} B_{\epsilon}(0)}\|x\|\|(I-P)(x /\|x\|)-\Lambda \Pi H(x /\|x\|)\| \\
=\epsilon \inf _{y \in \text { bdry } B_{1}(0)}\|(I-P) y-\Lambda \Pi H y\| .
\end{aligned}
$$

But $I-P-\Lambda \Pi H$ does not vanish on bdry $B_{1}(0)$ because every possible zero $y$ would be such that $y \in \operatorname{Ker} L, H y \in \operatorname{Im} L$ which, by (3.9), is only possible when $y=0$. Therefore, by a now classical argument [7],

with $\alpha>0$.

$$
\inf _{y \in \text { bdry } B_{1}(0)}\|(I-P) y-\Lambda \Pi H y\|=\alpha,
$$

Now, using (3.8) and (3.10), there exists $\rho_{1}>0$ such that $\mathrm{cl} B_{\rho_{1}}(0) \subset \Omega$ and

$$
\|\Lambda \Pi R x\| \leqslant(\alpha / 3)\|x\|^{k}, \quad\left\|L_{P}^{-1}(I-Q)(H+R) x\right\| \leqslant(\alpha / 3)\|x\|
$$

for every $x \in \operatorname{cl} B_{\rho_{1}}(0)$. Hence, for each $\left.\epsilon \in\right] 0, \rho_{1}\left[\right.$ and every $x \in$ bdry $B_{\epsilon}(0)$,

$$
\begin{aligned}
\left\|x-M_{2} x\right\| & \geqslant \inf _{x \in \text { bdry } B_{\epsilon}(0)}\|x-P x-\| x\left\|^{k-1} \Lambda \Pi H x\right\| \\
& -\sup _{x \in \text { bdry } B_{\epsilon}(0)}\|x\|^{1-k}\|\Lambda \Pi R x\| \\
& -\sup _{x \in \text { bdry } B_{\epsilon}(0)}\left\|L_{P}^{-1}(I-Q)(H+R) x\right\| \\
& -(1 / 3) \epsilon \alpha>0 .
\end{aligned}
$$

Thus 0 is an isolated coincidence point of $L$ and $N$ and $i[(L, N), 0]$ is defined. Furthermore, using (3.12), (3.13), Leray's product theorem and usual properties of Leray-Schauder's degree and index, we obtain

$$
\begin{aligned}
i[(L, N), 0] & =i_{L S}\left[I-M_{1}, 0\right] \cdot i_{L S}\left[I-M_{2}, 0\right] \\
& =d_{B}\left[(\epsilon I) \mid \operatorname{ker} L, B_{\epsilon}(0) \cap \operatorname{Ker} L, 0\right] \cdot i_{L S}\left[I-P-\|x\|^{1-k} \Lambda \Pi H, 0\right] \\
& =i_{B}\left[\left(I-P-\epsilon^{1-k} \Lambda \Pi H\right) \mid \operatorname{ker} L, 0\right]=i_{B}[-(\Lambda \Pi H) \mid \operatorname{Ker} L, 0],
\end{aligned}
$$

where the subscript $B$ indicates that we deal with a Brouwer degree or index [5] , [18]. If we still note that, using Proposition 3.1 of [9], $\Lambda \Pi H=J Q H$ with $J$ defined above, the proof is complete.

Now we shall suppose that $L: \operatorname{dom} L \subset X \rightarrow Z$ satisfies assumption $\left(\mathrm{H}_{1}\right)$, 
that $A: X \rightarrow Z$ is linear and continuous and, with notations of $\S 2$, that $B: \Omega$ $\rightarrow Z$ is continuous, $B(\Omega)$ is bounded, $L_{P}^{-1}(I-Q) A$ compact and $L_{P}^{-1}(I-Q) B$ completely continuous in $\Omega$. We shall also assume that $L^{\prime}=L-A$ : $\operatorname{dom} L \subset$ $X \rightarrow Z$ has a closed range with finite codimension and we shall denote by $Q^{\prime}$ a continuous projector such that $\operatorname{Ker} Q^{\prime}=\operatorname{Im} L^{\prime}$. We then have the following

LEMMA 3.3. If assumptions above hold for $L, A, B$ and $L^{\prime}$, then $\operatorname{ker} L^{\prime}$ is finite-dimensional and $\left(L_{P^{\prime}}^{\prime}\right)^{-1}\left(I-Q^{\prime}\right) B$ is completely continuous on $\Omega$, with $L_{P^{\prime}}^{\prime}=L^{\prime} \mid \operatorname{ker} P^{\prime}$ and $P^{\prime}$ is a continuous projector onto $\operatorname{ker} L^{\prime}$.

Proof. By Theorem 3.1 of [9], $\operatorname{ker} L^{\prime}=\operatorname{ker}\left(I-M_{\Lambda}^{*}\right)$ with $M_{\Lambda}^{*}=P+$ $\Lambda \Pi A+L_{P}^{-1}(I-Q) A$ and, from Riesz theory, is finite-dimensional. Let $P^{\prime}$ be a continuous projector onto ker $L^{\prime}$.

Hence, the mapping $\left(I-M_{\Lambda}^{*}\right) \mid \operatorname{ker} P^{\prime}: \operatorname{ker} P^{\prime} \rightarrow X$, which is clearly one-toone, is the sum of the linear homeomorphism $I \mid$ ker $P^{\prime}$ from $\operatorname{ker} P^{\prime}$ onto the closed subspace $\operatorname{ker} P^{\prime}$ of $X$ and of the compact mapping $-M_{\Lambda}^{*} \mid \operatorname{ker} P^{\prime}$. Using the extended Riesz theory (cf. for examples [3, p. 330, Problem 2]), $\left(I-M_{\Lambda}^{*}\right) \mid$ $\operatorname{ker} P^{\prime}$ is a linear homeomorphism of $\operatorname{ker} P^{\prime}$ onto $\left(I-M_{\Lambda}^{*}\right)\left(\operatorname{ker} P^{\prime}\right)$. On the other side, from Proposition 3.3 of [9] we have $I-M_{\Lambda}^{*}=\left[\Lambda \Pi+L_{P}^{-1}(I-Q)\right] L^{\prime}$ on dom $L$, with $\Lambda \Pi+L_{P}^{-1}(I-Q)$ an algebraic isomorphism of $Z$ onto dom $L$. Hence,

$$
L_{P^{\prime}}^{\prime}=L^{\prime} \mid \operatorname{ker} P^{\prime}=\left[\Lambda \Pi+L_{P}^{-1}(I-Q)\right]^{-1}\left[\left(I-M_{\Lambda}^{*}\right) \mid \operatorname{ker} P^{\prime}\right],
$$

which implies that the equality

$$
\left(L_{P^{\prime}}^{\prime}\right)^{-1}=\left[\left(I-M_{\Lambda}^{*}\right) \mid \operatorname{ker} P^{\prime}\right]^{-1}\left[\Lambda \Pi+L_{P}^{-1}(I-Q)\right]
$$

holds on $\operatorname{Im} L^{\prime}$, and, in particular,

$$
\begin{aligned}
\left(L_{P^{\prime}}^{\prime}\right)^{-1}\left(I-Q^{\prime}\right) B & =\left[\left(I-M_{\Lambda}^{*}\right) \mid \operatorname{ker} P^{\prime}\right]^{-1}\left[\Lambda \Pi+L_{P}^{-1}(I-Q)\right] B \\
& =\left[\left(I-M_{\Lambda}^{*}\right) \mid \operatorname{ker} P^{\prime}\right]^{-1}\left[\Lambda \Pi+L_{P}^{-1}(I-Q)\right] Q^{\prime} B .
\end{aligned}
$$

The first term in the right-hand member of this equality is clearly completely continuous on $\Omega$ and the same is true for the second term if we remark that a linear mapping is always continuous on a finite-dimensional space and if we use the fact that $B$ is continuous and $B(\Omega)$ is bounded.

We shall now use Lemmas 3.2 and 3.3 to prove a result which is complementary to Theorem 3.2 in that it gives a way to compute $i[(L, N), 0]$ when $N=A+B$ and $\operatorname{ker}(L-A) \neq\{0\}$.

For this purpose, let $L: \operatorname{dom} L \subset X \rightarrow Z$ satisfy assumption $\left(\mathrm{H}_{1}\right)$ and $N$ be of the form $N=A+H+R$ with $A: X \rightarrow Z$ be as in Lemma 3.3, $H: X \rightarrow$ $Z$ and $R: \Omega \rightarrow Z$ as in Lemma 3.2 and such that $L_{P}^{-1}(I-Q) H$ is completely continuous on $\operatorname{cl} B_{1}(0)$ and $L_{P}^{-1}(I-Q) N$ completely continuous on $\Omega$. Let us 
also suppose that $L^{\prime}=L-A$ is a Fredholm mapping of index zero, that $P^{\prime}, Q^{\prime}$, $L_{P^{\prime}}$ have the same meaning as in Lemma 3.3 and let us write $N^{\prime}=N-A=H+$ $R$. We have then the following

THEOREM 3.3. ( $L, N)$ and $\left(L^{\prime}, N^{\prime}\right)$ being defined as above and satisfying the quoted assumptions, let us suppose that $H x \notin \operatorname{Im} L^{\prime}$ for every $x \in \operatorname{ker} L^{\prime}$ such that $\|x\|=1$. Then 0 is an isolated zero for $L-N$ and

$$
i[(L, N), 0]=i_{B}\left[-\left(J^{\prime} Q^{\prime} H\right) \mid \operatorname{ker} L^{\prime}, 0\right] \cdot i\left[\left(L, A-\left(J^{\prime}\right)^{-1} P^{\prime}\right), 0\right]
$$

where $J^{\prime}=\Lambda^{\prime} \Pi^{\prime} \mid \operatorname{Im} Q^{\prime}$ with $\Pi^{\prime}: Z \rightarrow \operatorname{coker} L^{\prime}$ the canonical surjection and $\Lambda^{\prime}$ : coker $L^{\prime} \rightarrow \operatorname{ker} L^{\prime}$ an isomorphism preserving orientation chosen upon coker $L^{\prime}$ and $\operatorname{ker} L^{\prime}$.

Proof. From the assumptions and Lemma 3.3, it is easy to see that $\left(L^{\prime}, N^{\prime}\right)$ satisfies the assumptions of Lemma 3.2, which implies

$$
i\left[\left(L^{\prime}, N^{\prime}\right), 0\right]=i_{B}\left[-\left(J^{\prime} Q^{\prime} H\right) \mid \operatorname{ker} L^{\prime}, 0\right] .
$$

On the other hand, the way used in Theorem 1 of [10] can be applied here under the assumptions made and furnishes the relation

$$
i[(L, N), 0]=i\left[\left(L, A-\left(J^{\prime}\right)^{-1} P^{\prime}\right), 0\right] \cdot i\left[\left(L^{\prime}, N^{\prime}\right), 0\right],
$$

which, together with (3.15), gives the asked relation.

REMARK 3.1. If $\operatorname{ker}(L-A)=\{0\}$, it follows from Lemma 3.1 that $L^{\prime}=$ $L-A$ satisfies the needed assumptions and the supplementary condition upon $H$ is necessarily satisfied. In this case, (3.14) reduces exactly to (3.5) if we use the convention introduced in [10] for the Brouwer degree of a mapping in a 0-dimensional vector space.

The result of type (3.14) for Leray-Schauder index, first given by Krasnosel'skii [5] in a slightly different form and proved in a very complicated way, can be easily deduced from Theorem 3.3.

COROLlaRY 3.1. Let $A: X \rightarrow X$ be linear, compact and such that

$$
X=\operatorname{ker}(I-A) \oplus \operatorname{Im}(I-A),
$$

$H: X \rightarrow \bar{X}$ be completely continuous on $\operatorname{cl} B_{1}(0)$, homogeneous of degree $k>1$ and satisfying (3.8) and $R: \Omega \rightarrow X$ completely continuous and satisfying (3.10). Then, if $H x \notin \operatorname{Im}(I-A)$ for every $x \in \operatorname{ker}(I-A)$ such that $\|x\|=1$, and if $N=$ $A+H+R$, we have

$$
\begin{aligned}
i[(I, N), 0] & =i_{L S}[I-N, 0] \\
& =i_{B}\left[-\left(P^{\prime} H\right) \mid \operatorname{ker}(I-A), 0\right] \cdot i_{L S}[(I-A) \mid \operatorname{Im}(I-A), 0] \\
& =i_{B}\left[-\left(P^{\prime} H\right) \mid \operatorname{ker}(I-A), 0\right] \cdot i_{L S}[I-(1-\epsilon) A, 0]
\end{aligned}
$$


where $P^{\prime}: X \rightarrow X$ is a projector such that $\operatorname{Im} P^{\prime}=\operatorname{Ker}(I-A)$ and $\epsilon>0$ is sufficiently small.

Proof. By using the fact that every linear compact perturbation of identity is a continuous Fredholm mapping of index zero [1], it is easily checked that conditions of Theorem 3.3 are satisfied with $L^{\prime}=I-A$ and moreover we can take $P^{\prime}=Q^{\prime}$ and $J^{\prime}$ the identity in $\operatorname{ker}(I-A)$. On the other hand, it follows from (3.16) and Riesz theory that $\operatorname{Im} P^{\prime}$ and $\operatorname{ker} P^{\prime}$ are invariant for $I-A$ and hence

$$
\begin{aligned}
i\left[\left(I, A-P^{\prime}\right), 0\right] & =i_{L S}\left[I-A+P^{\prime}, 0\right] \\
& =i_{L S}\left[I-A\left(I-P^{\prime}\right), 0\right]=i_{L S}\left[(I-A) \mid \operatorname{ker} P^{\prime}, 0\right],
\end{aligned}
$$

which together with (3.14) gives (3.17). On the other hand, let us consider the homotopy

$$
I-M(\lambda)=I-A+\epsilon \lambda A+(1-\lambda) P^{\prime}, \quad \lambda \in[0,1],
$$

where $\epsilon>0$ is such that $\mu=1$ is the unique characteristic value of $A$ situated in $[1-\epsilon, 1+\epsilon]$. Clearly, $[I-M(1)] x=(I-(1-\epsilon) A) x \neq 0$ for $x \neq 0$. Moreover, $I-M(\lambda)=[(1-\lambda) I+\epsilon \lambda A] P^{\prime}+[I-(1-\epsilon \lambda) A]\left(I-P^{\prime}\right)$, the two operators of the right-hand member taking respectively their values in $\operatorname{Im} P^{\prime}$ and $\operatorname{ker} P^{\prime}$. Hence, if $x=M(\lambda) x$ for some $\lambda \in[0,1[$, we have

$$
x=P^{\prime} x, \quad P^{\prime} x+\epsilon \lambda(1-\lambda)^{-1} A P^{\prime} x=0
$$

which shows that $x=P^{\prime} x$ is an eigenvector of $A$ for the eigenvalue $-\epsilon \lambda(1-\lambda)^{-1}$. This number being different from 1 for each $\lambda \in[0,1[$, it follows from Riesz theory that $x$ must be zero. Hence,

$$
\begin{aligned}
i_{L S}\left[I-A+P^{\prime}, 0\right] & =i_{L S}[I-M(0), 0]=i_{L S}[I-M(1), 0] \\
& =i_{L S}[I-(1-\epsilon) A, 0]
\end{aligned}
$$

which implies (3.18).

For other papers devoted to the computation of Leray-Schauder index under various conditions, see $[14],[15],[19]$.

4. Characteristic values and their multiplicity for some couples of linear mappings. Let $X, Z$ be locally convex separated vector spaces on the complex field, $L:$ dom $L \subset X \rightarrow Z$ a linear mapping and $A: X \rightarrow Z$ a continuous linear mapping which satisfy the following assumptions:

$\left(\mathrm{H}_{1}^{\prime \prime}\right) L$ is a Fredholm mapping of the index zero;

$\left(\mathrm{H}_{2}^{\prime \prime}\right) L_{P}^{-1}(I-Q) A$ is compact,

where the notations are those of $\S 2$.

Definition 4.1. $\mu \in \mathrm{C}$ will be said to be a characteristic value for $(L, A)$ if $\operatorname{ker}(L-\mu A) \neq\{0\}$ and will be said to be a regular covalue for $(L, A)$ if $(L-\mu A)^{-1}$ exists on $Z$ and is continuous. 
These concepts have been introduced in a more general form in [6] and reduce respectively to the classical ones of characteristic value of $A$ and of the inverse of regular value for $A$ when $X=Z$ and $L=I$.

In the light of the equivalence theorem proved in [9], we obtain immediately

$$
\operatorname{ker}[L-\mu A]=\operatorname{ker}\left[I-M_{\Lambda}^{*}(\mu)\right]
$$

with

$$
M_{\Lambda}^{*}(\mu)=P+\mu \Lambda \Pi A+\mu L_{P}^{-1}(I-Q) A .
$$

This theorem was used to prove in [6] that, under assumptions $\left(\mathrm{H}_{1}^{\prime \prime}\right)-\left(\mathrm{H}_{2}^{\prime \prime}\right)$, $\mu \in \mathrm{C}$ is either a characteristic value or a regular covalue for $(L, A)$.

Moreover, with the same assumptions, we shall prove now a result about the structure of the set of characteristic values for $(L, A)$.

LEMMA 4.1. If assumptions $\left(\mathrm{H}_{1}^{\prime \prime}\right)-\left(\mathrm{H}_{2}^{\prime \prime}\right)$ hold, then the set of characteristic values for $(L, A)$ is either $\mathrm{C}$ or a set at most countable with only one possible accumulation point at infinity.

Proof. If there exists one regular covalue $\mu_{1}$ for $(L, A)$, we have, in the same way as in Lemma 3.1,

$$
\left[I-M_{\Lambda}^{*}(\mu)\right]=\left[I-M_{\Lambda}^{*}\left(\mu_{1}\right)\right]\left[I-\left(\mu-\mu_{1}\right)\left(L-\mu_{1} A\right)^{-1} A\right] .
$$

Then, if we note that the characteristic values for $(L, A)$ are just translates by $\mu_{1}$ of the classical ones for the compact mapping $\left[L-\mu_{1} A\right]^{-1} A$, the result follows at once from Riesz theory.

Thus in order to obtain a nontrivial spectral problem for $(L, A)$, we must add sufficient conditions on $(L, A)$ to insure that the characteristic values do not fill up C. That $\left(\mathrm{H}_{1}^{\prime \prime}\right)-\left(\mathrm{H}_{2}^{\prime \prime}\right)$ are not sufficient to prevent this "spectral degeneracy" is shown by taking

$$
X=Z=\mathrm{C}^{2}, \quad L:(x, y) \mapsto(x, 0) \text { and } A:(x, y) \mapsto(y, 0) .
$$

But before looking for those auxiliary conditions, we will consider a second useful aspect of a spectral theory, the notion of multiplicity associated to a characteristic value.

In fact, since in general the space $X$ differs from the space $Z$, the classical definition of multiplicity of a characteristic value for a linear compact perturbation of the identity fails. On the other side, from the equivalence theorems of [9], it would be natural to relate the multiplicity of a characteristic value for $(L, A)$ to the dimension of $\operatorname{ker}\left[I-M_{\Lambda}^{*}(\mu)\right]^{n_{0}}$, with $n_{0}$ the smallest integer such that $\operatorname{ker}\left[I-M_{\Lambda}^{*}(\mu)\right]^{n_{0}+1}=\operatorname{ker}\left[I-M_{\Lambda}^{*}(\mu)\right]^{n_{0}}$. Such a number exists by the compactness of $M_{\Lambda}^{*}(\mu)$ but, unfortunately, it depends upon the (usually nonunique) choice of projectors $P$ and $Q$, as shown in the following example. 
EXAMPLE 4.1. Let $X=Z=C^{2}$,

Then

$$
L:(x, y) \mapsto(0,-x+y), \quad A:(x, y) \mapsto(-y,-x+y) .
$$

$$
L-\mu A:(x, y) \mapsto[\mu y,(1-\mu)(y-x)]
$$

has the characteristic values $\mu_{1}=0$ and $\mu_{2}=1$. On the other hand,

and hence

$$
\operatorname{ker} L=\{(x, x), x \in \mathrm{C}\}, \quad \operatorname{Im} L=\{(0, x), x \in \mathrm{C}\}
$$

$$
P:(x, y) \mapsto(x, x), \quad Q_{1}:(x, y) \mapsto(x, x), \quad Q_{2}:(x, y) \mapsto(x, 0)
$$

are respectively such that the sequences

$$
\mathrm{C}^{2} \stackrel{P}{\longrightarrow} \mathrm{C}^{2} \stackrel{L}{\longrightarrow} \mathrm{C}^{2} \stackrel{Q_{i}}{\longrightarrow} \mathrm{C}^{2}, \quad i=1,2,
$$

are exact and it is easily computed that $L_{P}^{-1}:(0, x) \mapsto(0, x)$. By taking respectively $J_{i}=\Lambda \Pi \mid \operatorname{Im} Q_{i}, i=1,2$ as follows:

$$
\begin{aligned}
& J_{1}: \mid \operatorname{Im} Q_{1} \rightarrow \operatorname{ker} L,(x, x) \mapsto(x, x), \\
& J_{2}: \mid \operatorname{Im} Q_{2} \rightarrow \operatorname{ker} L,(x, 0) \mapsto(x, x),
\end{aligned}
$$

we see at once that the mappings $M_{i}^{*}(\mu)=P+\mu J_{i} Q_{i} A+\mu L_{P}^{-1}\left(I-Q_{i}\right) A$ $(i=1,2)$ are such that, for $\mu=1$,

$$
I-M_{1}^{*}(1):(x, y) \mapsto(y, 0), \quad I-M_{2}^{*}(1):(x, y) \mapsto(y, y) .
$$

Hence we obtain

$$
\operatorname{ker}\left[I-M_{i}^{*}(1)\right]=\operatorname{ker}[L-A]=\{(x, 0), x \in X\}, \quad i=1,2,
$$

but unfortunately,

$$
\begin{aligned}
& \operatorname{ker}\left[I-M_{1}^{*}(1)\right]^{2}=C^{2} \neq \operatorname{ker}\left[I-M_{1}^{*}(1)\right], \\
& \operatorname{ker}\left[I-M_{2}^{*}(1)\right]^{2}=\operatorname{ker}\left[I-M_{2}^{*}(1)\right] .
\end{aligned}
$$

The considerations above lead to introducing supplementary assumptions on $(L, A)$ both to insure the spectral "nondegeneracy" and make possible a reasonable definition of the multiplicity of a characteristic value for $(L, A)$. For this purpose, let us suppose from now on that,

$\left(\mathrm{H}_{3}^{\prime \prime}\right)$ for every $x \in \operatorname{ker} L /\{0\}, A x \notin \operatorname{Im} L$, and let us prove a number of preliminary results, beginning with the following basic

Proposition 4.1. If assumptions $\left(\mathrm{H}_{1}^{\prime \prime}\right)-\left(\mathrm{H}_{3}^{\prime \prime}\right)$ hold, there exists a (unique) continuous projector $Q_{A}: Z \rightarrow Z$ such that

$$
\operatorname{Im} Q_{A}=A(\operatorname{ker} L), \quad \operatorname{ker} Q_{A}=\operatorname{Im} L .
$$


Proof. By condition $\left(\mathrm{H}_{3}^{\prime \prime}\right), A \mid$ ker $L$ is one-to-one and hence, from $\left(\mathrm{H}_{1}^{\prime \prime}\right)$,

$$
\operatorname{dim} A(\operatorname{ker} L)=\operatorname{dim} \operatorname{ker} L=\operatorname{codim} \operatorname{Im} L
$$

which, together with the fact that $A(\operatorname{ker} L) \cap \operatorname{Im} L=\{0\}$, implies that $A(\operatorname{ker} L)$ is an algebraic, and hence a topological, supplement of $\operatorname{Im} L$ in $Z$ [16]. The existence and uniqueness of $Q_{A}$ follow at once [16].

REMARK 4.1. An immediate but important consequence of Proposition 4.1 , is that, for every $u \in \operatorname{ker} L$,

$$
\left(I-Q_{A}\right) A u=0 .
$$

As we do not emphasize here the dependence of $M_{\Lambda}^{*}(\mu)$ with respect to $\Lambda$ and that the corresponding mapping $M_{\Lambda}^{*}(\mu)$ with $Q=Q_{A}$ will play a basic role in what follows, we shall denote it, to avoid heavy notations, by $M_{A}$. More generally, we shall write, for every $\mu \in \mathbf{C}$,

$$
\begin{aligned}
M_{A}(\mu) & =P+\mu \Lambda \Pi A+\mu L_{P}^{-1}\left(I-Q_{A}\right) A, \\
D(\mu) & =I-P-\mu \Lambda \Pi A .
\end{aligned}
$$

The following "factorization theorem" is essential for what follows.

Proposition 4.2. If assumptions $\left(\mathrm{H}_{1}^{\prime \prime}\right)-\left(\mathrm{H}_{3}^{\prime \prime}\right)$ are satisfied, then, for each $\mu \in \mathbf{C}$,

$$
I-M_{A}(\mu)=\left[I-\mu L_{P}^{-1}\left(I-Q_{A}\right) A\right] \cdot D(\mu) .
$$

Moreover, $D(\mu)$ is a linear homeomorphism for every $\mu \neq 0$.

Proof. The first part of the proof follows at once from Remark 4.1 and a simple calculation. To prove the second part, we remark that $D(\mu)$ is a compact perturbation of identity with, if $\mu \neq 0$, a kernel given by $\{x \in X: x \in$ ker $L, \Pi A x=0$ \}, i.e., a trivial one by using assumption $\left(\mathrm{H}_{3}^{\prime \prime}\right)$. Hence, from the Fredholm alternative [16], $D(\mu)$ is a linear homeomorphism for each nonzero $\mu$.

This result makes it possible to associate an ordinary characteristic value problem for compact linear mappings to the spectral problem for $(L, A)$.

Definition 4.2. If assumptions $\left(\mathrm{H}_{1}^{\prime \prime}\right)-\left(\mathrm{H}_{3}^{\prime \prime}\right)$ hold, a spectral operator associated to $(L, A)$ will be an operator of the form $L_{P}^{-1}\left(I-Q_{A}\right) A$, with $P: X$ $\rightarrow X$ any continuous projection onto $\operatorname{ker} L$.

The following results justify the terminology of Definition 4.2.

Proposition 4.3. If assumptions $\left(\mathrm{H}_{1}^{\prime \prime}\right)-\left(\mathrm{H}_{3}^{\prime \prime}\right)$ hold, the set of nonzero characteristic values for $(L, A)$ is equal to the set of characteristic values of $L_{P}^{-1}\left(I-Q_{A}\right) A$, with $P$ any projector in $X$ onto ker $L$. In particular, the characteristic values for $(L, A)$ form a set at most countable with only one possible accumulation point at infinity. 
Proof. The first part of the proposition is an easy consequence of Proposition 4.2 and the second one of the compactness of $L_{P}^{-1}\left(I-Q_{A}\right) A$ and Riesz theory.

The proposition above shows that assumptions $\left(\mathrm{H}_{1}^{\prime \prime}\right)-\left(\mathrm{H}_{3}^{\prime \prime}\right)$ not only insure the "spectral nondegeneracy" for $(L, A)$ but give precise information, clearly independent of the choice of the projector $P$, about the localization of the characteristic values for $(L, A)$. That this result is strongly related to the choice of the particular projector $Q_{A}$ is shown by the following example proving that, with $Q_{A}$ replaced by an arbitrary projector $Q$, the first assertion of Proposition 4.3 can already be false.

EXAMPLE 4.2. Let $X=Z=\mathrm{C}^{2}$.

$$
L:(x, y) \mapsto(0, y) \text { and } A:(x, y) \mapsto(x-y, x-y) .
$$

Thus

$$
L-\mu A:(x, y) \mapsto(\mu(y-x), y+\mu(y-x))
$$

and 0 is the unique characteristic value for $(L, A)$.

On the other side, we have $Q_{A}:(x, y) \mapsto(x, x)$ and, by taking $P=Q$ : $(x, y) \mapsto(x, 0)$ the sequence $\mathrm{C}^{2} \stackrel{P}{\longrightarrow} \mathrm{C}^{2} \stackrel{L}{\longrightarrow} \mathrm{C}^{2} \stackrel{Q}{\longrightarrow} \mathrm{C}^{2}$ is exact. Then, by an easy calculation, we obtain, for any $\mu \in \mathbf{C}$

$$
\begin{aligned}
I-\mu L_{P}^{-1}(I-Q) A:(x, y) & \mapsto(x, y-\mu(x-y)), \\
I-\mu L_{P}^{-1}\left(I-Q_{A}\right) A:(x, y) & \mapsto(x, y) .
\end{aligned}
$$

The first operator $L_{P}^{-1}(I-Q) A$ has the characteristic value $\mu=-1$, but the second one $L_{P}^{-1}\left(I-Q_{A}\right) A$ has no characteristic value, in harmony with Proposition 4.3.

We shall now come back to the problem of defining a multiplicity in the light of assumption $\left(\mathrm{H}_{3}^{\prime \prime}\right)$. First, we will study the dependence with respect to $P$ of the multiplicity of a characteristic value of $L_{P}^{-1}\left(I-Q_{A}\right) A$ and, for brevity we shall denote respectively by $K_{A}$ and $K_{A}^{\prime}$ the operators $L_{P}^{-1}\left(I-Q_{A}\right) A$ and $L_{P^{\prime}}^{-1}\left(I-Q_{A}\right) A$ with $P, P^{\prime}$ two projectors onto ker $L$.

Proposition 4.4. If assumptions $\left(\mathrm{H}_{1}^{\prime \prime}\right)-\left(\mathrm{H}_{3}^{\prime \prime}\right)$ hold and if $P, P^{\prime}$ are continuous projectors onto ker $L$, then the respective multiplicities of $\mu$ as a characteristic value of $K_{A}$ and $K_{A}^{\prime}$ are equal.

Proof. Let us first note that, by the form of $K_{A}$ and $K_{A}^{\prime}$ and Remark 4.1, Im $P$ and ker $P$ (resp. $\operatorname{Im} P^{\prime}$ and ker $P^{\prime}$ ) are invariant subspaces for $I-\mu K_{A}$ (resp. $I-\mu K_{A}^{\prime}$ ) and that $I-\mu K_{A}$ (resp. $I-\mu K_{A}$ ) acts as the identity on $\operatorname{Im} P$ (resp. $\operatorname{Im} P^{\prime}$ ). Therefore we have, for each positive integer $n$, 


$$
\begin{aligned}
\left(I-\mu K_{A}\right)^{n} x & =\left(I-\mu K_{A}\right)^{n-1}\left[P x+\left(I-\mu K_{A}\right)(I-P) x\right] \\
& =\cdots=P x+\left(I-\mu K_{A}\right)^{n}(I-P) x
\end{aligned}
$$

and a corresponding relation with $\boldsymbol{P}^{\prime}$ instead of $P$. This shows that,

$$
\begin{aligned}
& \operatorname{ker}\left(I-\mu K_{A}\right)^{n}=\operatorname{ker}\left[\left(I-\mu K_{A}\right)^{n} \mid \operatorname{ker} P\right], \\
& \operatorname{ker}\left(I-\mu K_{A}^{\prime}\right)^{n}=\operatorname{ker}\left[\left(I-\mu K_{A}^{\prime}\right)^{n} \mid \operatorname{ker} P^{\prime}\right] .
\end{aligned}
$$

On the other hand, using Proposition 2.2 of [9] and (4.2), we have

$$
\begin{aligned}
\left(I-P^{\prime}\right)\left(I-\mu K_{A}\right)^{n} & =\left(I-P^{\prime}-\mu K_{A}^{\prime}\right)\left(I-\mu K_{A}\right)^{n-1} \\
& =\left(I-\mu K_{A}^{\prime}\right)\left(I-P^{\prime}\right)\left(I-\mu K_{A}\right)^{n-1} \\
& =\cdots=\left(I-\mu K_{A}^{\prime}\right)^{n}\left(I-P^{\prime}\right) .
\end{aligned}
$$

Therefore, using (4.7), (4.8) and the fact that $\left(I-P^{\prime}\right) \mid$ ker $P$ is a linear homeomorphism of ker $P$ onto ker $P^{\prime}$ we see easily that $\operatorname{ker}\left(I-\mu K_{A}\right)^{n}$ and $\operatorname{ker}\left(I-\mu K_{A}^{\prime}\right)^{n}$ are isomorphic for each positive integer $n$ and Proposition 4.4 follows then at once from the definition of the multiplicity.

Now let $\mu$ be a characteristic value for $(L, A), \nu(\mu)$ the smallest integer such that

$$
\operatorname{ker}\left[I-M_{A}(\mu)\right]^{n+1}=\operatorname{ker}\left[I-M_{A}(\mu)\right]^{n}, \quad n \geqslant \nu(\mu),
$$

and $\nu^{\prime}(\mu)$ the multiplicity of $\mu$ as a characteristic value of a compact linear operator $K_{A}$ associated to $(L, A)$.

We have the following basic

Proposition 4.5. If assumptions $\left(\mathrm{H}_{1}^{\prime \prime}\right)-\left(\mathrm{H}_{3}^{\prime \prime}\right)$ hold, then, for every nonzero characteristic value $\mu$ for $(L, A)$ we have $\nu(\mu)=\nu^{\prime}(\mu)$ and

$$
\operatorname{dim} \operatorname{ker}\left[I-M_{A}(\mu)\right]^{\nu(\mu)}=\operatorname{dim} \operatorname{ker}\left(I-\mu K_{A}\right)^{\nu^{\prime}(\mu)} .
$$

Proof. Let us first prove that, for each $\mu \in \mathbf{C} \backslash\{0\}$, and each positive integer $n$,

$$
\operatorname{Im}\left[I-M_{A}(\mu)\right]^{n}=\operatorname{Im}\left(I-\mu K_{A}\right)^{n} .
$$

By (4.5), $I-M_{A}(\mu)=\left(I-\mu K_{A}\right) D(\mu)$, and, by Proposition 4.2, $D(\mu)$ is a linear homeomorphism of $X$ having, by assumption $\left(\mathrm{H}_{3}^{\prime \prime}\right)$, a restriction to $\operatorname{ker} L$ which is an automorphism of ker $L$. Hence, from the form of $D(\mu)$, we have $D(\mu)(\operatorname{ker} L \oplus Y)=\operatorname{ker} L \oplus Y$ for every vector subspace $Y$ of $\operatorname{ker} P$. Using now (4.6) with $n=1$, we have

$$
\operatorname{Im}\left(I-\mu K_{A}\right)=\operatorname{ker} L \oplus \operatorname{Im}\left[\left(I-\mu K_{A}\right) \mid \operatorname{ker} P\right]
$$


with $\operatorname{Im}\left[\left(I-\mu K_{A}\right) \mid \operatorname{ker} P\right] \subset$ ker $P$. Therefore, if $n$ is a positive integer,

$$
\begin{aligned}
\operatorname{Im}\left[I-M_{A}(\mu)\right]^{n} & =\left[I-M_{A}(\mu)\right]^{n-1}\left(I-\mu K_{A}\right) D(\mu)(X) \\
& =\left[I-M_{A}(\mu)\right]^{n+1}\left[\operatorname{Im}\left(I-\mu K_{A}\right)\right] \\
& =\left[I-M_{A}(\mu)\right]^{n-2}\left(I-\mu K_{A}\right) D(\mu)\left\{\operatorname{ker} L \oplus \operatorname{Im}\left[\left(I-\mu K_{A}\right) \mid \operatorname{ker} P\right]\right\} \\
& =\left[I-M_{A}(\mu)\right]^{n-2}\left(I-\mu K_{A}\right)\left\{\operatorname{ker} L \oplus \operatorname{Im}\left[\left(I-\mu K_{A}\right) \mid \operatorname{ker} P\right]\right\} \\
& =\left[I-M_{A}(\mu)\right]^{n-2} \operatorname{Im}\left(I-\mu K_{A}\right)^{2}=\cdots=\operatorname{Im}\left(I-\mu K_{A}\right)^{n} .
\end{aligned}
$$

Now, it follows at once from (4.10) that the numbers $\nu(\mu)$ and $\nu^{\prime}(\mu)$, whose existence is insured by Riesz theory, are necessarily equal. Moreover, the finite-dimensional spaces $\operatorname{ker}\left[I-M_{A}(\mu)\right]^{\nu(\mu)}$ and $\operatorname{ker}\left(I-\mu K_{A}\right)^{\nu(\mu)}$ are such that

$$
\begin{aligned}
X & =\operatorname{Ker}\left[I-M_{A}(\mu)\right]^{\nu(\mu)} \oplus \operatorname{Im}\left[I-M_{A}(\mu)\right]^{\nu(\mu)} \\
& =\operatorname{ker}\left(I-\mu K_{A}\right)^{\nu(\mu)} \oplus \operatorname{Im}\left(I-\mu K_{A}\right)^{\nu(\mu)},
\end{aligned}
$$

which, together with (4.10), implies (4.9).

We are now able to introduce the following

DEFinition 4.3. If assumptions $\left(\mathrm{H}_{1}^{\prime \prime}\right)-\left(\mathrm{H}_{3}^{\prime \prime}\right)$ hold, the multiplicity $\beta(\mu)$ of the characteristic value $\mu \in \mathbf{C}$ for $(L, A)$ is the number

$$
\beta(\mu)=\operatorname{dim} \operatorname{ker}\left[I-M_{A}(\mu)\right]^{\nu(\mu)} .
$$

That $\beta(\mu)$ uniquely depends upon $L, A$ and $\mu$ is insured by Propositions 4.4 and 4.5. When $X=Z, L=I$ and $A$ is compact, $\beta(\mu)$ is nothing but the classical multiplicity of the characteristic value $\mu$ of $A$.

Moreover, it follows from Propositions 4.4 and 4.5 that the multiplicity of any nonzero characteristic value $\mu$ for $(L, A)$ is equal to the (classical) multiplicity of $\mu$ as characteristic value of any compact linear operator $K_{A}$ associated to $(L, A)$. On the other side, 0 is a characteristic value for $(L, A)$ if and only if $\operatorname{ker} L \neq\{0\}$ and, from the fact that $I-M_{A}(0)=I-P$, it is clear that $\nu(0)=1$ and

$$
\beta(0)=\operatorname{dim}[\operatorname{ker}(I-P)]=\operatorname{dim} \operatorname{ker} L .
$$

Another justification of the interest of Definitions 4.2 and 4.3 will be found in the following section.

\section{A generalization of Leray-Schauder's formula for the index of linear} mappings. We shall now use the concepts and results of $\S \S 2$ and 4 to extend, in the frame of the coincidence index theory, the famous Leray-Schauder formula quoted above. In this section, $X$ and $Z$ will be normed real vector spaces, $L$ : $\operatorname{dom} L \subset X \rightarrow Z$ will be linear, $A: X \rightarrow Z$ linear and continuous, and $(L, A)$ will be supposed throughout to satisfy assumptions $\left(\mathrm{H}_{1}^{\prime \prime}\right)-\left(\mathrm{H}_{3}^{\prime \prime}\right)$ of $\S 4$. 
THEOREM 5.1. If $\mu \in \mathbf{R}$ is not a characteristic value for $(L, A)$, then (5.1) $i[(L, \mu A), 0]=(-1)^{\gamma}(\operatorname{sign} \mu)^{\operatorname{dim} k e r L} \cdot \operatorname{sign} \operatorname{dét}[-(\Lambda \Pi A) \mid \operatorname{ker} L]$, where $\gamma$ is the sum of the multiplicities of the real characteristic values for $(L, A)$ strictly situated between 0 and $\mu$, and where $\operatorname{sign} \operatorname{det}[-(\Lambda \Pi A) \mid \operatorname{ker} L]=$ +1 if $\operatorname{ker} L=\{0\}$.

Proof. Using formula (4.5) and the fact that $\mu$ is not a characteristic value for $(L, A)$, each mapping in (4.5) is a linear homeomorphism and hence, using Leray's product theorem we obtain

$$
i[(L, \mu A), 0]=i_{L S}\left[I-\mu K_{A}, 0\right] \cdot i_{L S}[D(\mu), 0] .
$$

By the Leray-Schauder theorem relating the index and the multiplicities and by Proposition 4.5, we have $i_{L S}\left(I-\mu K_{A}, 0\right)=(-1)^{\gamma}$ and, on the other side,

$$
\begin{aligned}
i_{L S}(D(\mu), 0) & =i_{B}[-\mu(\Lambda \Pi A) \mid \operatorname{ker} L, 0]=+1 \text { or } \\
& =(\operatorname{sign} \mu)^{\operatorname{dim} \operatorname{Ker} L} \cdot \operatorname{sign} \operatorname{dét}[-(\Lambda \Pi A) \operatorname{ker} L]
\end{aligned}
$$

according to $\operatorname{Ker} L=\{0\}$ or $\operatorname{ker} L \neq\{0\}$. Formula (5.1) follows at once from (5.2).

If $X=Z, L=I$ and $A$ is compact, Theorem 4.1 just reduces to LeraySchauder's result. It is also possible to give a "relative" form of (5.1) which is more elegant.

COROLlaRY 5.1. Under the assumptions above, if the real numbers $\mu<$ $\mu^{\prime}$ are not characteristic values for $(L, A)$, then

$$
i\left[\left(L, \mu^{\prime} A\right), 0\right]=(-1)^{\delta} i[(L, \mu A), 0],
$$

where $\delta$ is the sum of the multiplicities of the characteristic values for $(L, A)$ lying in $] \mu, \mu^{\prime}[$.

Proof. From (5.1) applied to $\mu$ and $\mu^{\prime}$ we obtain easily, with obvious notations,

$$
i\left[\left(L, \mu^{\prime} A\right), 0\right]=(-1)^{\gamma^{\prime}-\gamma} \operatorname{sign}\left(\mu \mu^{\prime}\right)^{\operatorname{dim} \operatorname{ker} L_{i}[(L, \mu A), 0] .}
$$

Now, if $\mu \mu^{\prime}>0, \gamma^{\prime}-\gamma$ is equal to $\delta$ and (5.3) follows at once. If $\mu \mu^{\prime}<0, \delta=$ $\gamma+\beta(0)+\gamma^{\prime}=\gamma+\operatorname{dim} \operatorname{Ker} L+\gamma^{\prime}$ by using (4.12) and hence

$$
(-1)^{\gamma^{\prime}-\gamma} \operatorname{sign}\left(\mu \mu^{\prime}\right)^{\operatorname{dim} \operatorname{ker} L}=(-1)^{\gamma^{\prime}+\gamma+\operatorname{dim} \operatorname{ker} L}=(-1)^{\delta}
$$

and the proof is complete.

As an application of Corollary 5.1 , let $(L, A)$ be a couple of mappings satisfying assumptions $\left(\mathrm{H}_{1}^{\prime \prime}\right)-\left(\mathrm{H}_{2}^{\prime \prime}\right)$ and let us suppose that there exists a real characteristic value $\mu^{\prime}$ for $(L, A)$ such that the mapping $L^{\prime}=L-\mu^{\prime} A$ satisfies the conditions (whose notations are obvious), 
$\left(\mathrm{H}_{1}^{\prime \prime}\right) L^{\prime}$ is a Fredholm mapping of index zero.

$\left(\mathrm{H}_{3}^{\prime \prime \prime}\right)$ For every $x \in \operatorname{ker} L^{\prime} \backslash\{0\}, A x \notin \operatorname{Im} L^{\prime}$.

Then we have the following

CoROllary 5.2. If assumptions $\left(\mathrm{H}_{1}^{\prime \prime}\right)-\left(\mathrm{H}_{2}^{\prime \prime}\right)$ and $\left(\mathrm{H}_{1}^{\prime \prime}\right)-\left(\mathrm{H}_{3}^{m}\right)$ hold, then,

(a) the set of characteristic values for $(L, A)$ is at most a countable set with only one possible accumulation point at infinity;

(b) for every $\epsilon>0$ such that $\mu^{\prime}$ is the unique characteristic value lying in $\left[\mu^{\prime}-\epsilon, \mu+\epsilon\right]$, we have

$$
i\left[\left(L,\left(\mu^{\prime}+\epsilon\right) A\right), 0\right]=(-1)^{\operatorname{dim} \operatorname{ker} L^{\prime}} i\left[\left(L,\left(\mu^{\prime}-\epsilon\right) A\right), 0\right] .
$$

Proof. (a) From the obvious equality $L-\mu A=L^{\prime}-\left(\mu-\mu^{\prime}\right) A$, we see that the characteristic values for $(L, A)$ are just translates by $\mu^{\prime}$ of the ones for $\left(L^{\prime}, A\right)$. On the other hand, using Lemma 3.3, $\left(L_{P^{\prime}}^{\prime}\right)^{-1}\left(I-Q^{\prime}\right) A$ is compact. Hence, using Proposition 4.3 for $\left(L^{\prime}, A\right)$, we obtain the desired property.

(b) By a direct application of Corollary 5.1 and (4.12) we have

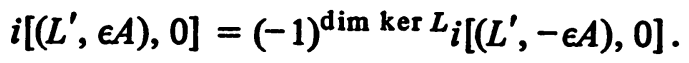

But, using the argument of Theorem 1 of [10], we obtain

$$
\begin{aligned}
i\left[\left(L,\left(\mu^{\prime}+\epsilon\right) A\right), 0\right] & =\gamma_{0} \cdot i\left[\left(L^{\prime}, \epsilon A\right), 0\right], \\
i\left[\left(L,\left(\mu^{\prime}-\epsilon\right) A\right), 0\right] & =\gamma_{0} \cdot i\left[\left(L^{\prime},-\epsilon A\right), 0\right]
\end{aligned}
$$

where $\gamma_{0}=i\left[\left(L,-\left(J^{\prime}\right)^{-1} P^{\prime}+\mu^{\prime} A\right), 0\right]$ and $J^{\prime}$ is some isomorphism between $\operatorname{Im} Q_{A}^{\prime}$ and $\operatorname{ker} L^{\prime}$. (5.5) then follows at once from (5.6) and (5.7).

We can also give the following complement to Theorem 3.2.

COROLlaRY 5.3. If assumptions of Theorem 3.2 and condition $\left(\mathrm{H}_{3}^{n}\right)$ hold, then

$$
i[(L, N), 0]=(-1)^{\gamma} \operatorname{sign} \operatorname{dét}[-(\Lambda \Pi A) \mid \operatorname{ker} L]
$$

where $\gamma$ is the sum of the multiplicities of the characteristic values for $(L, A)$ lying in ]0, 1 [.

REMARK 5.1. $\$ \S 4$ and 5 have emphasized the importance of assumption $\left(\mathrm{H}_{3}^{n}\right)$ which leads to select the crucial projector $Q_{A}$ to define the multiplicity. If one considers relation (5.3) in the equivalent form

$$
\begin{aligned}
i[(L, \mu A), 0]= & \operatorname{sign}\left(\mu \mu^{\prime}\right)^{\operatorname{dim} \operatorname{ker} L} \cdot i_{L S}\left[I-\mu L_{P}^{-1}\left(I-Q_{A}\right) A, 0\right] \\
& \cdot i\left[\left(L, \mu^{\prime} A\right), 0\right],
\end{aligned}
$$

and if one remembers that the coincidence index is independent of the choice of the projectors $P$ and $Q$, one could expect that (5.9) remains true by replacing $Q_{A}$ by an arbitrary $Q$. This is of course not the case if we refer to Example 
4.2 showing that a characteristic value for $L_{P}^{-1}(I-Q) A$ is not necessarily a characteristic value for $L_{P}^{-1}\left(I-Q_{A}\right) A$ when $Q \neq Q_{A}$.

REMARK 5.2. It will be useful to check that, in Example 4.1, condition $\left(\mathrm{H}_{3}^{\prime \prime}\right)$ is satisfied and that $Q_{2}=Q_{A}$, so that the various phenomena and results developed in $\$ \S 4$ and 5 can be explicitly illustrated by this example.

REMARK 5.3. Striking applications of the results of this paper can be easily found in the theory of bifurcations for equations of the form $L x-N(x, \mu)=0$, when $L$ and $N$ satisfy assumptions of Theorems 3.2 or 3.3. More precisely, applications of Theorem 3.2 have been given by the first author in [6] while the use of Theorem 3.3 can easily extend this type of result to cases where the linear part of $L-N(\cdot, \mu)$ is not invertible. Lastly, the results of $\$ 5$ can be useful in comparing coincidence indices associated to different regular covalues for $(L, A(\cdot)$ ), a technique which is fundamental in bifurcation theory (cf. for examples $[5],[6])$.

\section{REFERENCES}

1. R. A. Bonic, Linear functional analysis, Notes on Math. and its Applications, Gordon and Breach, New York and London, 1969. MR 41 \#2336.

2. F. E. Browder, Non-linear functional equations in locally convex spaces, Duke Math. J. 24 (1957), 579-589. MR 19, 1184.

3. J. Dieudonné, Eléments d'analyse. Tome I: Fondements de l'analyse moderne, 2nd rev. ed., Cahiers Scientifiques, fasc. 28, Gauthiers-Villars, Paris, 1968. MR 38 \#4246.

4. J. K. Hale and J. Mawhin, Periodic solutions of neutral equations, J. Differential Equations 15 (1974), 295-307.

5. M. A. Krasnosel'ski1, Topological methods in the theory of nonlinear integral equations, GITTL, Moscow, 1956; English transl., Macmillan, New York, 1964. MR 20 \#3464; 28 \#2414.

6. B. Laloux, Indice de coïncidence et bifurcations, Equations Différentielles et Fonctionnelles non Linéaires, P. Janssens, J. Mawhin and N. Rouche, Editors, Hermann, Paris, 1973, pp. 109-121.

7. J. Leray and J. Schauder, Topologie et équations fonctionnelles, Ann. Sci. École Norm. Sup. (3) 51 (1934), 45-78.

8. J. Leray, Topologie des espaces abstraits de M. Banach, C. R. Acad. Sci. Paris 200 (1935), $1082-1084$.

9. J. Mawhin, Equivalence theorems for nonlinear operator equations and coincidence degree theory for some mappings in locally convex topological vector spaces, J. Differential Equations 12 (1972), 610-636. MR 48 \#7045.

10. - A further invariance property of coincidence degree in convex spaces, Ann. Soc. Sci. Bruxelles Sér. I 87 (1973), 51-57. MR 47 \#9372.

11. The solvability of some operator equations with a quasi-bounded nonlinearity in normed spaces, J. Math. Anal. Appl 45 (1974), 455-467. MR 48 \#12187.

12. Periodic solutions of some vector retarded differential equations, $J$. Math. Anal. Appl. 45 (1974), 588-603. MR 48 \#11725.

13. - Problémes aux limites du type de Neumann pour certaines équations différentielles ou aux dérivées partielles non linéaires, Equations Différentielles et Fonctionnelles non Linéaires, P. Janssens, J. Mawhin and N. Rouche, Editors, Hermann, Paris, 1973, pp. 123-134.

14. V. B. Melamed, On the computation of the index of the fixed point of a compact vector field, Dokl. Akad. Nauk SSSR 126 (1959), 501-504. (Russian) MR 22 \#12415.

15. On the computation of the rotation of a compact vector field in the critical case, Sibirsk. Mat. Ž. 2 (1961), 414-427. (Russian) MR 24 \#A2227. 
16. A. P. Robertson and W. J. Robertson, Topological vector spaces, Cambridge Tracts in Math. and Math. Physics, no. 53, Canıbridge Univ. Press., New York, 1964. MR 28 \#5318.

17. N. Rouche and J. Mawhin, Equations différentielles ordinaires, Vol. II, Masson, Paris, 1973.

18. J. T. Schwartz, Nonlinear functional analysis, Gordon and Breach, New York, 1969.

19. P. P. Zabrelko and M. A. Krasnosel'skii, Calculation of the index of a fixed point of a vector field, Sibirsk. Mat. Ž. 5 (1964), 509-531; English transl., Amer. Math. Soc. Transl. (2) 56 (1966), 273-295. MR 29 \#3893.

20. F. E. Browder, Topology and non-linear functional equations, Studia Math. 31 (1968), 189-204. MR 38 \#6410.

INSTITUT MATHEMATIQUE, UNIVERSITE DE LOUVAIN, B-1348, LOUVAIN-LANEUVE, BELGIUM 